\title{
Revisión de la literatura sobre logística inversa, sus aplicaciones y tendencias futuras
}

\section{(Literature Review of Reverse Logistic, applications and future trends)}

\author{
Jorge Campoverde ${ }^{1}$, Martha Elena Carrillo², Jonnathan Jiménez Yumbla³, Raúl Roldán Nariño 4 , \\ Diego Loyola ${ }^{5}$ y Katherine Coronel-Pango/ ${ }^{6}$
}

\section{Resumen}

En el artículo se presenta una revisión de la literatura sobre investigaciones, casos de aplicación y exploración de logística inversa (LI). Para ello, se utilizaron varias bases de datos para la recuperación de los artículos. La revisión ha permitido analizar los conceptos iniciales de la LI y complementarlos con nuevas ópticas y argumentos desarrollados por varios autores. El principal aporte es la taxonomía propuesta en el desarrollo del artículo, conformada por la relación de temas y términos encontrados en la revisión literaria. Se han identificado los campos de aplicación más importantes y las tendencias futuras en materia de LI, en donde se ha determinado que es necesario ampliar el análisis de la LI hacia una perspectiva económica, social, cultural y ambiental.

\section{Palabras clave}

Logística inversa, taxonomía, cadena cerrada

\begin{abstract}
This article presents a review of the literature on Reverse Logistics $(R L)$ research, application cases and exploration. Various databases were used to retrieve articles. This review has allowed to analyze initial concepts of $R L$ and complementary ones with new optics and arguments developed by various authors. The main contribution is the taxonomy proposed in the development of the article. It was made up of relationship of themes and terms found in the literary review. Also, the most important fields of application and future trends in RL have been identified, where it has been determined that it is necessary to extend the analysis of RL towards an economic, social, cultural and environmental perspective.
\end{abstract}

\section{Keywords}

Reverse logistic, taxonomy, closed loop chain.

\footnotetext{
$1 \quad$ Universidad de Cuenca. Cuenca, Ecuador. [jorge.campoverde@ucuenca.edu.ec]

2 Pontificia Universidad Javeriana de Colombia, Bogotá, Colombia. [mcarrillajaveriana.edu.co]

3 Universidad de Cuenca. Cuenca, Ecuador. [jonnathan.jimenezy@ucuenca.edu.ec]

4 Pontificia Universidad Javeriana de Colombia. Bogotá, Colombia. [rroldan@javeriana.edu.co]

5 Universidad de Cuenca. Cuenca, Ecuador. [diego.loyola@ucuenca.edu.ec]

6 Universidad de Cuenca. Cuenca, Ecuador. [katherine.coronelp95@ucuenca.edu.ec]
} 


\section{Introducción}

Una de las primeras definiciones de LI fue la presentada ante el Council of Logistic Management por J. Stock (1992), quien la definió como

... el término utilizado a menudo para referirse al papel de la logística en el reciclaje, la eliminación de residuos y la gestión de materiales peligrosos; una perspectiva más amplia incluye todo lo relacionado con las actividades logísticas llevadas a cabo en la reducción de fuentes, reciclaje, sustitución, reutilización de materiales y eliminación.

Carter y Ellram (1998) realizaron uno de los primeros trabajos académicos en el campo de la LI. En su estudio recogen las primeras aproximaciones a los conceptos de LI. La LI no debe ser considerada simplemente como un proceso de recuperación de materiales o productos, sino que se la debe incorporar en un proceso de reducción de materiales desde su fabricación, de manera tal que los insumos que se utilicen en el proceso de producción también se reduzcan y fluyan en menor cantidad hacia atrás.

La Reverse Logistic y Sustainability Organization (2007) definió a la LI como "el proceso de mover bienes de su destino final típico a otro punto, con el propósito de capturar valor que de otra manera no estaría disponible, para la disposición apropiada de los productos". En su estudio, De Brito y Dekker (2014) indican que a la LI o reversa se la conocía con otros nombres, tales como: logística de retorno, logística retro o distribución reversa, sin embargo, la discrepancia de términos ha sido una de las causas por las que no ha existido un consenso sobre el alcance y la definición de la LI. Por esta razón, no se debe confundir la LI con la gestión de residuos. La gestión de residuos hace referencia únicamente a la recolección y eliminación de residuos, es decir, productos para los que no es factible un nuevo uso, mientras que la LI contempla fases de reutilización, reprocesamiento y reciclaje. Van Engeland et al. (2018) explican que en la literatura científica coexisten dos grandes áreas: eliminación atractiva y la gestión de los residuos. La LI no es simétricamente opuesta a la logística directa. La diferencia entre LI y logística directa puede interpretarse mediante varios atributos como la cantidad, categoría, tiempo de ciclo, unidad de mantenimiento de inventario y rutas de distribución. Los productos devueltos suelen ser pequeños en cantidad y tienen muchos tipos diferentes.

Stock (1992) dice que la LI requiere inversión y que los costos varían entre el 4 \% y el $9.49 \%$ del total de los costos logísticos de la empresa. En el caso del sector del retail, los costos de aplicar LI oscilan entre el 5 \% y 6 \%. Para Mihi-Ramírez et al. (2012), la LI debe ser considerada como una oportunidad de inversión para las empresas y no como un gasto, pues la empresa debe perseguir el incremento de sus beneficios, aumentar el valor de la marca, anticiparse a la demanda y mejorar la detección de necesidades de los clientes. De esta manera, explica Srivastava (2008), se consigue un mayor porcentaje de retención y atracción de la clientela. De Brito y Dekker (2014) indican que las principales razones para hacer LI son beneficios económicos, disposiciones legales y presiones de los clientes asociada a la creciente cultura ciudadana respecto a la recuperación de residuos y productos. Asimismo, Mar y Gracia (2015) consideran que los beneficios de la LI se clasifican en dos grupos: beneficios por la recuperación de productos y beneficios por la recuperación de envases. Mihi-Ramírez et al. (2012) indica que las causas por las cuales en las empresas españolas es necesario iniciar un proceso de LI son las siguientes: producto dañado o no deseado (25.38 \%), errores en pedidos o insatisfacción del cliente (20.63\%), fin de la vida útil del producto (20.10\%), exceso de inventario (11.7\%), acuerdos contractuales (8.13 \%), fin de promoción (6.14 \%), ajustes de inventario (4.92 \%) y reciclaje (3 \%). 
Desde 1992, cuando Stock propuso uno de los primeros conceptos, se han desarrollado un sinnúmero de trabajos en el marco de la LI, desde diferentes ópticas y de manera técnica, a través del desarrollo de modelos matemáticos que han pretendido dar soluciones de optimización en el campo ambiental analizando el impacto que tienen los desechos y proponiendo opciones de recuperación de materiales; y desde el campo social como una forma de posicionamiento de marca de las empresas, entre otros.

En general, el impacto y las aristas que ha generado y está generando la LI son amplias. En este sentido, los aspectos en los que los autores se han enfocado son los siguientes: diseño de la cadena y ruteo (Li et al., 2017; Ranjbaran et al., 2019; Govindan et al., 2020; Mahmoudi \& Parviziomran, 2020), optimización a través de modelos matemáticos (Zarbakhshnia et al., 2020), planificación (Govindan et al., 2020), economía circular (Donner et al., 2020; Julianelli et al., 2020; Zarbakhshnia et al., 2020), entre otros. Cabe recalcar que la mayor parte de producción científica está disponible en inglés, y los estudios se los ha desarrollado en países europeos o asiáticos. Se ha encontrado muy poca evidencia científica de procesos de LI que se hayan llevado o se están llevando a cabo en América Latina. Por ejemplo, algunos trabajos desarrollados en Colombia, Venezuela, Brasil, Cuba y Argentina corresponden a las primeras aproximaciones que se han hecho en materia de LI.

A pesar de los amplios estudios, existe una falta de convergencia de conceptos sobre la LI y su aplicación, por lo que el objetivo propuesto para este artículo es analizar la literatura existente para identificar la taxonomía de dichos conceptos en función de los cuales se pueda determinar las tendencias y futuras líneas de investigación en esta temática. También se plantea un acápite con un análisis centrado en Latinoamérica, aspecto que generalmente no se considera en los análisis revisados.

\section{Metodología}

El análisis literario se ha hecho a partir de la revisión de 2457 artículos académicos indexados en Web of Science, Scopus, Latindex y Scielo, lo que ha permitido constatar que los autores convergen en algunos términos comunes. Se ha considerado una temporalidad de 10 años, es decir, desde el 2010 hasta el 2020. La ecuación de búsqueda ha sido reverse logistic o 'logística inversa' utilizada tanto en los títulos como en las palabras clave. A partir de las palabras clave, se ha construido la taxonomía, la cual permite identificar claramente los conceptos y orientar las líneas de investigación. Para construir la taxonomía, se ha basado en la literatura y en estudios previos en los que se ha identificado tres grandes áreas de análisis con respecto a la LI.

Con el fin de encontrar los temas que los investigadores han mencionado en sus escritos científicos al tratar la LI, se revisaron 9030 palabras claves (solo proporcionadas por el autor) de 2457 artículos, de las cuales se han tomado las más representativas, así como su evolución. Por último, se analiza detalladamente la producción en América del Sur considerando la misma metodología planteada.

\section{Resultados}

La ecuación de búsqueda reverse logistic o 'logística inversa' para documentos en una ventana de tiempo desde 2010 hasta 2020 y con un alcance geográfico de todo el mundo muestra un crecimiento sostenido del número de artículos a partir del año 2015 (Figura 1). Esta tendencia 
de crecimiento es posible explicarla con un incremento en la percepción que el tema ha tomado día a día, porque se ha incrementado la relevancia en el mundo ante la concienciación ambiental creciente. Aunque a principios de la década parece notarse que decrecía el interés en el tema, a medida que transcurre el tiempo de análisis, se descubre una clara tendencia de aumento.

Figura 1. Cantidad de documentos publicados por año

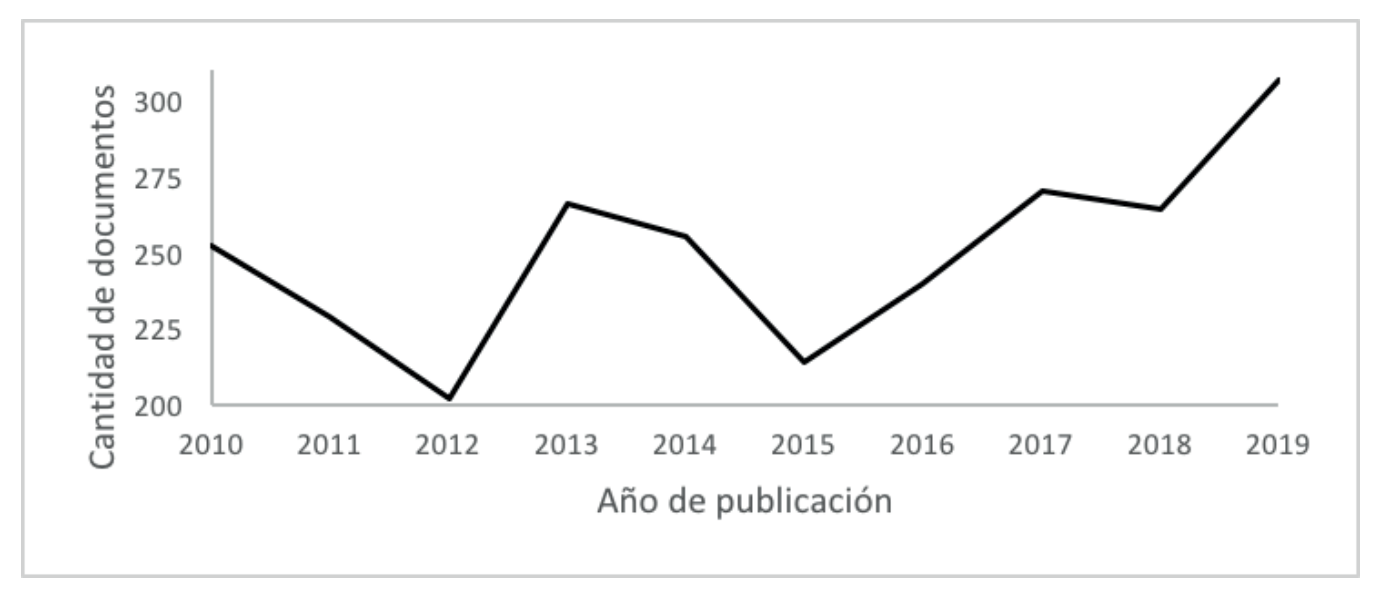

En los últimos diez años, algunos autores han hecho valiosas contribuciones al tema de LI. Como se puede apreciar en la tabla 1, se detalla el autor y el número de trabajos relacionados con la LI, de manera que se puede ver, por ejemplo, que Kannan Govindan es uno de los autores que mayor aporte ha desarrollado, algunos de manera individual, mientras que otros los ha desarrollado junto con otros investigadores.

Tabla 1. Autores y número de contribuciones en LI en los últimos 10 años

\begin{tabular}{|c|c|c|c|}
\hline Autor & Documentos & Autor & Documentos \\
\hline Govindan Kannan & 32 & Wang Y. & 13 \\
\hline Li Y. & 18 & Jha P.C. & 12 \\
\hline Zhang Y. & 16 & Agrawal Saurabh & 12 \\
\hline Zhang G. & 15 & Shankar R. & 11 \\
\hline S. H. & 13 & Hazen B.T. & 11 \\
\hline Singh R.K. & 13 & Wang X. & 11 \\
\hline Diabat A. & 13 & Chileshe N. & 11 \\
\hline Jaber M.Y. & 13 & Rameezdeen R. & \\
\hline LiX. & 13 & & \\
\hline
\end{tabular}

La mayoría de los trabajos de estos autores han sido revisados y son pilares fundamentales en el desarrollo de este trabajo, entre estos se destacan Kazemi et al. (2019), quienes hicieron una revisión bibliométrica de los artículos publicados en la revista International Journal of Production of Research7 sobre la LI. Govindan, Agarwal, et al. (2019) analizaron las empresas tecnológicas indias en las cuales se propone una red de cadena de suministro integrada para

$7 \quad$ Una de las principales revistas en difundir investigaciones relacionadas con este tema. 
la evaluación y la selección de socios directos y de proveedores externos de la cadena de la LI, para lo cual se utiliza un proceso de jerarquía analítica difusa, con una programación de enteros mixtos de dos objetivos, para maximizar el beneficio del fabricante y el puntaje sostenible de los proveedores. Govindan et al. (2016) desarrollaron un modelo en el que se considera integralmente aspectos ambientales, sociales y económicos, así como los indicadores. Este modelo fue desarrollado a través de optimización multiobjetivo, multiperiodo y multienfoque para construir una red de logística sostenible. Reddy et al. (2020) plantean un modelo de programación lineal de enteros mixtos para resolver una red de LI verde de varios periodos y varios niveles incluyendo la selección del tipo de vehículo. En general se puede apreciar el carácter técnico de estos trabajos, además de su aplicación en países asiáticos y europeos, pero también la falta de trabajos relacionados con la LI en América, especialmente América del Sur.

Se han resumido las palabras claves utilizadas por los autores (tabla 2), incluyendo los autores que los mencionan, lo cual ha permitido construir un concepto propio que se presenta a continuación:

Logística inversa es la cadena cerrada que abarca el proceso o conjunto de estrategias a través de las cuales los flujos retornan al inicio, produciendo la devolución de productos o materiales, que posteriormente se reciclan, reutilizan, reparan, remanufacturan, recolectan 0 renuevan, con el objetivo de recuperar valor, contribuir con el medio ambiente. Este procedimiento constituye una ventaja competitiva en la gestión empresarial, al conseguir eficiencia y optimización de costos, equipos y rutas, lo cual se logra aplicando modelos matemáticos, con el enfoque de redes de suministro verdes y el diseño de redes de LI.

Tabla 2. Palabras más mencionadas y autores

\begin{tabular}{|c|c|}
\hline Palabra Clave & Autores \\
\hline Remanufacturing & (Alamerew \& Brissaud, 2020; Reddy et al., 2020) \\
\hline Sustainability & $\begin{array}{l}\text { (Agrawal et al., 2016b; Govindan et al., 2016; Mangla et al., 2016; } \\
\text { Govindan, Agarwal, et al., 2019; Govindan, Kadziński, et al., 2019; } \\
\text { Liu et al., 2019; Zarbakhshnia et al., 2020; Julianelli et al., 2020) }\end{array}$ \\
\hline Supply chain management (SCM) & $\begin{array}{l}\text { (Agrawal et al., 2016a; Govindan et al., 2017; Van Engeland et al., } \\
\text { 2018; Kazemi et al., 2019) }\end{array}$ \\
\hline Recycling & $\begin{array}{l}\text { (Silva Rodríguez, 2017; Liu et al., 2019; Aydin, 2020; Guarnieri et al., } \\
\text { 2020; Karagoz et al., 2020; Wang et al., 2020) }\end{array}$ \\
\hline Closed-loop supply chain (SC) & $\begin{array}{c}\text { (Amin \& Baki, 2017; Misni \& Lee, 2017; Amin, et al., 2017; Heydari et al., 2017; } \\
\text { Kazemi et al., 2019; Govindan et al., 2020) }\end{array}$ \\
\hline Reverse logistic & $\begin{array}{l}\text { (Agrawal et al., 2016c; Mangla et al., 2016; Heydari et al., 2017; Amin et al., } \\
\text { 2017; Govindan \& Bouzon, 2018; Tosarkani \& Amin, 2018; Van Engeland et al., } \\
\text { 2018; Agrawal \& Singh, 2019; Govindan, Jha, et al., 2019; Herrera-Granda et al., } \\
\text { 2019; Kazemi et al., 2019; Guarnieri et al., 2020; Mahmoudi \& Parviziomran, } \\
\text { 2020; Reddy et al., 2020; Wang et al., 2020) }\end{array}$ \\
\hline Supply chain & (Batarfi et al., 2017; Heydari et al., 2017; Kazemi et al., 2019) \\
\hline Waste management & $\begin{array}{l}\text { (Van Engeland et al., 2018; Agrawal \& Singh, 2019; Donner et al., 2020; Kara- } \\
\text { goz et al., 2020; Mukherjee et al., 2020; Rossit et al., 2020) }\end{array}$ \\
\hline Network design & $\begin{array}{l}\text { (Govindan et al., 2017; Banguera et al., 2018; Ranjbaran et al., 2019; } \\
\text { Aydin, 2020; Govindan et al., 2020; Reddy et al., 2020) }\end{array}$ \\
\hline
\end{tabular}




\begin{tabular}{|c|c|}
\hline Green supply chain management & (Saffar et al., 2015; Misni \& Lee, 2017; Rabbani et al., 2017) \\
\hline Circular economy & $\begin{array}{l}\text { (Banguera et al., 2018; Agrawal \& Singh, 2019; Alamerew \& Brissaud, 2020; } \\
\text { Donner et al., 2020; Govindan et al., 2020; Guarnieri et al., 2020; } \\
\text { Jin et al., 2020; Julianelli et al., 2020) }\end{array}$ \\
\hline Genetic algorithm & $\begin{array}{l}\text { (Govindan et al., 2017; Tosarkani \& Amin, 2018; Govindan, Kadziński, } \\
\text { et al., 2019; Rossit et al., 2020; Trochu et al., 2020) }\end{array}$ \\
\hline Logistics & (Agrawal et al., 2016a; Ranjbaran et al., 2019) \\
\hline Optimization & $\begin{array}{l}\text { (Govindan, Paam et al., 2016; Tosarkani \& Amin, 2018; Herrera-Granda } \\
\text { et al., 2019; Trochu et al., 2020; Zarbakhshnia et al., 2020; Aydin, 2020) }\end{array}$ \\
\hline Uncertain & $\begin{array}{c}\text { (Ranjbaran et al., 2019; Zarbakhshnia et al., 2020; Aydin, 2020; } \\
\text { Rossit et al., 2020) }\end{array}$ \\
\hline $\begin{array}{l}\text { waste of electrical y electronic } \\
\text { equipment (WEEE) }\end{array}$ & $\begin{array}{c}\text { (Agrawal, et al. 2016c; Govindan, Agarwal, et al., 2019; } \\
\text { Rodrigues et al., 2020) }\end{array}$ \\
\hline Closed loop supply chain & (Agrawal et al., 2016c; Fattahi \& Govindan, 2017; Julianelli et al., 2020) \\
\hline Green supply chain & (Saffar et al., 2015; Misni \& Lee, 2017; Rabbani et al., 2017) \\
\hline Simulation & $\begin{array}{l}\text { (Fattahi \& Govindan, 2017; Alamerew \& Brissaud, 2020; } \\
\text { Rossit et al., 2020; Trochu et al., 2020) }\end{array}$ \\
\hline e-waste & $\begin{array}{l}\text { (Donner et al., 2020; Mukherjee et al., 2020; Rodrigues et al., } \\
\text { 2020; Trochu et al., 2020) }\end{array}$ \\
\hline
\end{tabular}

La LI es considerada por muchos autores como una cadena cerrada de suministro, es decir, es el proceso que complementa la logística directa y cierra los ciclos o bucles del proceso logístico. Otro de los aspectos importantes es el de la cadena verde de suministro, porque normalmente se relaciona la LI con el medio ambiente. En varios trabajos sobre la LI se muestra la aplicación e implementación de modelos matemáticos, de manera que se pretende dar soluciones ya sea por medio de procedimientos estocásticos o no estocásticos, lógicos o difusos a problemas propios de la LI. En este sentido, se desarrollan diseño de redes de logística, diseño de rutas, optimización y simulación, entre otros. Todos estos aspectos han permitido construir la taxonomía que se desarrolla en el siguiente apartado.

\subsection{Taxonomía de la LI}

Luego de revisar las 9030 palabras claves, se han tomado las más representativas (tabla 2) que se puede ver en la figura 2 , en donde se indica el número de veces que la palabra ha sido utilizada y la evolución por año. Al revisar estas 20 palabras claves sin ningún tipo de agrupamiento, es posible ver cómo las 11 primeras presentan unas frecuencias de uso relativamente más altas que el resto, donde resaltan principalmente un grupo relacionado con las tendencias de procesamiento como remanufactura, reciclado y manejo de desechos. Otro grupo de términos que muestra una importancia en su frecuencia de uso reúne tendencias en las estrategias empresariales en cuanto al manejo de la LI como sostenibilidad, cadenas de ciclo cerrado, diseño de redes, logística verde o economía circular. 
Figura 2. Relación de palabras claves por año

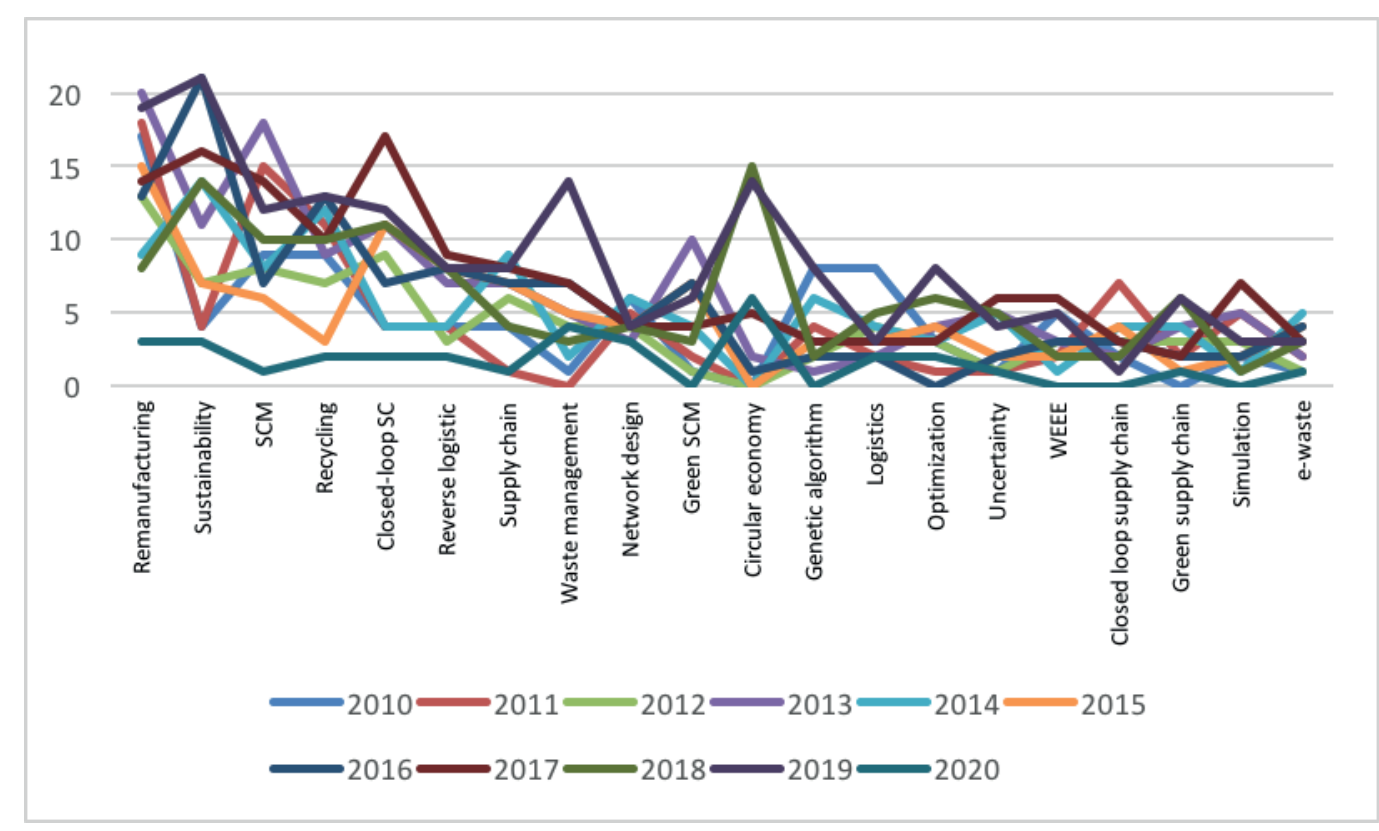

En estos dos grupos hay incrementos particulares en palabras como remanufactura, SCM, ciclo o cadena cerrada, gestión de desechos y economía circular, y se puede constatar las tendencias particulares en determinados años por las cuales los investigadores se inclinan para acercarse a un tema específico. A continuación, en la tabla 3, se detallan las frecuencias y porcentajes de la figura 2, lo que permite concluir una estrecha relación entre remanufactura y $\mathrm{LI}$, pues de todas las palabras es la de mayor uso. También resaltan sostenibilidad y SCM.

Tabla 3. Palabras claves por año de publicación

\begin{tabular}{|c|c|c|c|c|c|c|c|c|c|c|c|c|c|}
\hline Palabra clave & 2010 & 2011 & $\mathbf{2 0 1 2}$ & $\mathbf{2 0 1 3}$ & $\mathbf{2 0 1 4}$ & $\mathbf{2 0 1 5}$ & $\mathbf{2 0 1 6}$ & $\mathbf{2 0 1 7}$ & $\mathbf{2 0 1 8}$ & $\mathbf{2 0 1 9}$ & $\mathbf{2 0 2 0}$ & Total & $\%$ \\
\hline Remanufacturing & 17 & 18 & 13 & 20 & 9 & 15 & 13 & 14 & 8 & 19 & 23 & 149 & $12.57 \%$ \\
\hline Sustainability & 4 & 4 & 7 & 11 & 14 & 7 & 21 & 16 & 14 & 21 & 23 & 122 & $10.30 \%$ \\
\hline $\begin{array}{c}\text { Supply chain } \\
\text { management }\end{array}$ & 9 & 15 & 8 & 18 & 8 & 6 & 7 & 14 & 10 & 12 & 11 & 108 & $9.11 \%$ \\
\hline Recycling & 9 & 11 & 7 & 9 & 12 & 3 & 13 & 10 & 10 & 13 & 23 & 99 & $8.35 \%$ \\
\hline $\begin{array}{c}\text { Closed-loop } \\
\text { supply chain }\end{array}$ & 4 & 4 & 9 & 11 & 4 & 11 & 7 & 17 & 11 & 12 & 22 & 92 & $7.76 \%$ \\
\hline Reverse logistic & 4 & 4 & 3 & 7 & 4 & 8 & 8 & 9 & 8 & 8 & 12 & 65 & $5.49 \%$ \\
\hline Supply chain & 4 & 1 & 6 & 7 & 9 & 7 & 7 & 8 & 4 & 8 & 11 & 62 & $5.23 \%$ \\
\hline Waste management & 1 & 0 & 4 & 5 & 2 & 5 & 7 & 7 & 3 & 14 & 24 & 52 & $4.39 \%$ \\
\hline Network design & 6 & 5 & 4 & 3 & 6 & 4 & 4 & 4 & 4 & 4 & 13 & 47 & $3.97 \%$ \\
\hline $\begin{array}{c}\text { Green supply chain } \\
\text { management }\end{array}$ & 1 & 2 & 1 & 10 & 4 & 7 & 7 & 4 & 3 & 6 & 10 & 45 & $3.80 \%$ \\
\hline Circular economy & 0 & 0 & 0 & 2 & 0 & 0 & 1 & 5 & 15 & 14 & 16 & 43 & $3.63 \%$ \\
\hline Genetic algorithm & 8 & 4 & 2 & 1 & 6 & 3 & 2 & 3 & 2 & 8 & 10 & 39 & $3.29 \%$ \\
\hline Logistics & 8 & 2 & 4 & 2 & 4 & 3 & 2 & 3 & 5 & 3 & 12 & 38 & $3.21 \%$ \\
\hline Optimization & 3 & 1 & 3 & 4 & 3 & 4 & 0 & 3 & 6 & 8 & 12 & 37 & $3.12 \%$ \\
\hline
\end{tabular}




\begin{tabular}{|c|c|c|c|c|c|c|c|c|c|c|c|c|c|}
\hline Uncertainty & 1 & 1 & 1 & 5 & 5 & 2 & 2 & 6 & 5 & 4 & 11 & 33 & $2.78 \%$ \\
\hline $\begin{array}{c}\text { waste of electronic } \\
\text { equipment }\end{array}$ & 5 & 2 & 3 & 3 & 1 & 2 & 3 & 6 & 2 & 5 & 10 & 32 & $2.70 \%$ \\
\hline $\begin{array}{c}\text { Closed loop } \\
\text { supply chain }\end{array}$ & 2 & 7 & 3 & 2 & 4 & 4 & 3 & 3 & 2 & 1 & 10 & 31 & $2.62 \%$ \\
\hline Green supply chain & 0 & 2 & 3 & 4 & 4 & 1 & 2 & 2 & 6 & 6 & 11 & 31 & $2.62 \%$ \\
\hline Simulation & 2 & 5 & 3 & 5 & 1 & 2 & 2 & 7 & 1 & 3 & 10 & 31 & $2.62 \%$ \\
\hline e-waste & 1 & 2 & 1 & 2 & 5 & 4 & 4 & 3 & 3 & 3 & 11 & 29 & $2.45 \%$ \\
\hline Total & 89 & 90 & 85 & 131 & 105 & 98 & 115 & 144 & 122 & 172 & 285 & 1.427 & \\
\hline$\%$ & $6.23 \%$ & $6.31 \%$ & $5.96 \%$ & $9.18 \%$ & $7.36 \%$ & $6.87 \%$ & $8.06 \%$ & $10.09 \%$ & $8.54 \%$ & $12.05 \%$ & $19.97 \%$ & & \\
\hline
\end{tabular}

Las palabras claves analizadas (tabla 2 y figura 2) han servido como insumo para construir la taxonomía (clasificación de temáticas de LI) que permitió organizar y orientar este estudio. Esta clasificación surge de un procesamiento de las palabras para eliminar errores de la base de datos como comas, espacio, comillas y agrupar términos que se referían a una misma categoría. Empezando por el título, se agruparon los conceptos LI y revisión de literatura. En un segundo nivel de análisis se construyeron tres subgrupos, dependientes conceptualmente del tema revisión de literatura de LI.

1. Subgrupo técnicas de análisis: formado por 6 palabras clave.

2. Subgrupo procesos: fue creado por los autores para reunir una serie de 9 palabras.

3. Subgrupo logística integrada: formado por 13 palabras claves incluyendo el título del subgrupo (figura 3).

Figura 3. Taxonomía de la LI

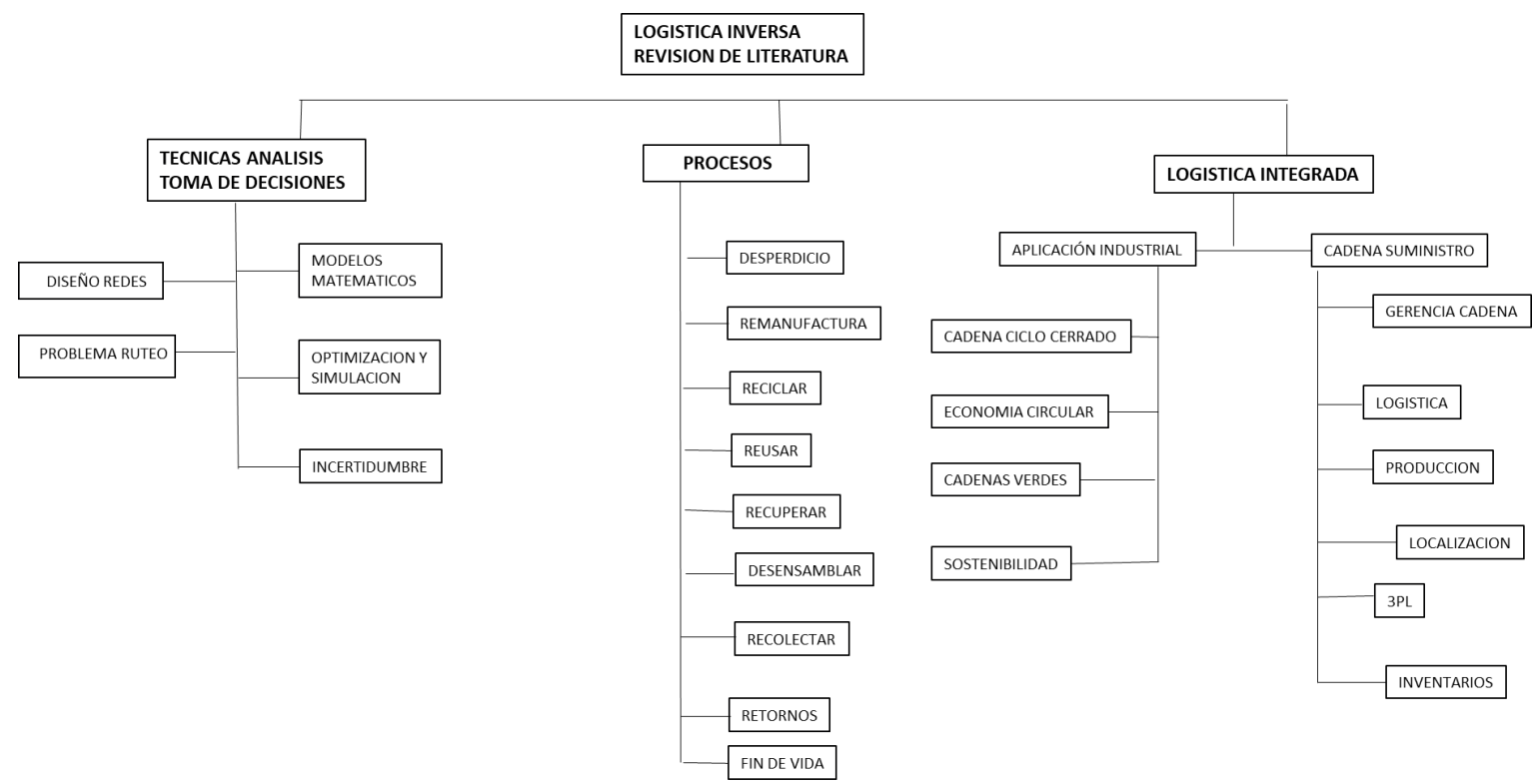

El primer subgrupo (técnicas de análisis) agrupa herramientas principalmente de tipo analítico matemático que aportan en la exploración de los posibles modelos analíticos usados 
en este tema. Entre ellos encontramos conceptos como modelos matemáticos, optimización y simulación, incertidumbre y problema de ruteo que son típicos estudios de esta categoría.

El segundo subgrupo, denominado procesos, incluye básicamente todas las categorías de formas de procesamiento que se pueden hacer en el momento de ejecutar logística en reversa. Adicionalmente incluye las palabras como desperdicio, retornos y fin de vida, propias de este análisis, que constituyen formas de describir algunos de los resultados obtenidos en la circulación de productos.

El tercer subgrupo, denominado logística integrada, involucra a su vez dos aspectos: aplicación industrial y cadena de suministro. El primero de ellos incluye una serie de aspectos relacionados con estrategias que son posibles asumir para diseñar cadenas de suministro ya sean directas o inversas. El segundo implica una serie de conceptos muy relacionados con la logística tradicional como localización, 3PL o inventarios, que también son necesarios para la LI.

\subsection{Aplicaciones de la LI en América Latina}

Para analizar la aplicación de la LI en América Latina, se ha realizado una búsqueda de "aplicación de logística inversa América Latina" y "reverse logistic application Latin America" en Google Schoolar, donde se encontraron 530 documentos, los cuales han sido revisados y resumidos en la tabla 4.

Tabla 4. Estudios por país

\begin{tabular}{|c|c|c|c|}
\hline $\begin{array}{l}\text { País de } \\
\text { América }\end{array}$ & Documentos & Porcentaje & Autores \\
\hline Brasil & 239 & $76.85 \%$ & $\begin{array}{c}\text { (Gutberlet et al., 2017; Bouzon et al., 2018; Abuabara et al., 2019; } \\
\text { Voigt et al., 2019; De Oliveira et al., 2019; Rodrigues et al., 2020; } \\
\text { De Oliveira \& Borges, 2020; Guarnieri et al., 2020; } \\
\text { Peña-Montoya et al., 2020) }\end{array}$ \\
\hline Colombia & 23 & $7.40 \%$ & $\begin{array}{c}\text { (Peña-Montoya et al., 2015; Silva Rodríguez, 2017; De La Hoz et al., } \\
\text { 2017; Gómez Montoya et al., 2017; Oyola \& Amaya-Mier, 2019, } \\
\text { marzo 5-7) }\end{array}$ \\
\hline México & 22 & $7.07 \%$ & $\begin{array}{l}\text { (Cruz-Netro et al., 2016; Pérez-Salazar et al., 2017; } \\
\text { Uriarte-Miranda et al., 2018; Piyathanavong et al., 2019) }\end{array}$ \\
\hline Chile & 8 & $2.57 \%$ & $\begin{array}{c}\text { (Johnson et al., 2015; Sepúlveda-Rojas \& Benitez-Fuentes, 2016, } \\
\text { mayo 10-14; Soto et al., 2016; Banguera et al., 2018; } \\
\text { Lagos et al., 2018) }\end{array}$ \\
\hline Argentina & 5 & $1.61 \%$ & $\begin{array}{c}\text { (Noé, 2015; Dondo \& Méndez, 2016; Gutberlet et al., 2017; } \\
\text { Rossit et al., 2020) }\end{array}$ \\
\hline Ecuador & 4 & $1.29 \%$ & $\begin{array}{l}\text { (Sepúlveda \& Banguera, 2017; Banguera et al., 2018; } \\
\text { Herrera-Granda et al., 2019; López et al, 2019, julio 24-26) }\end{array}$ \\
\hline Venezuela & 2 & $0.64 \%$ & (Bustos, 2015; García-Rodríguez et al., 2013) \\
\hline Perú & 1 & $0.32 \%$ & (Rusel \& Tello, 2019) \\
\hline Uruguay & 1 & $0.32 \%$ & (Rossit et al., 2020) \\
\hline
\end{tabular}

Se puede apreciar que Brasil es el país que lidera estudios en este tema con el 77 \% aproximadamente de los artículos publicados. Entre los estudios llevados a cabo en Brasil, Rodrigues 
et al. (2020) analizaron el flujo doméstico de los desechos electrónicos en Sao Paulo e indican que es complejo, debido a su generación difusa y la dependencia del comportamiento del consumidor. El objetivo del estudio pretendió apoyar desde la academia las políticas públicas relacionadas. Bouzon et al. (2018) evaluaron la interrelación entre las barreras logísticas inversas desde la perspectiva de las partes interesadas en el contexto brasileño, y determinaron que en el entorno brasileño existen aproximadamente 10 de 13 barreras que impiden la implementación adecuada de la LI.

En Colombia, Silva Rodríguez (2017) destaca la importancia de la LI en la recolección y acopio de desechos. Silva Rodríguez desarrolló un estudio con el objetivo de resolver el problema generado por la no devolución y recolección de envases de plaguicidas en el distrito de riego de Usochicamocha (Departamento de Boyacá, Colombia), para lo cual diseñó una red de LI, la cual contempla fases como recolección, acopio y disposición final de los residuos y recipientes de plaguicidas. De La Hoz et al. (2017) elaboró un modelo de programación lineal para la LI del sector de plástico polipropileno en una empresa de la zona industrial de Cartagena dedicada al proceso y comercialización de plásticos reciclados, en la cual realizaron un análisis para optimizar los factores relevantes que influyen a lo largo de la LI de toda la cadena del proceso asociado con la recuperación del plástico.

En 2015, en Córdoba (Argentina) se estudiaron 6 empresas a través de un enfoque cualitativo. Se pudo conocer que las empresas que habían incorporado en su planificación estratégica aspectos formales de LI, principalmente relacionados con el marco de acciones de sustentabilidad o responsabilidad social, obtenían un desempeño superior relacionado con principios no sólo económicos, sino también sociales y ambientales. Además, aquellas empresas que lo hicieron lograron ventajas competitivas con respecto a las que no lo hicieron (Noé, 2015). En Bahía Blanca (Argentina) y en Montevideo (Uruguay), Rossit et al. (2020) llevaron a cabo una aplicación experimental en escenarios reales de un problema de multioptimización que tuvo como objetivo optimizar la accesibilidad al sistema, la cantidad de residuos recolectados y el costo de instalación del manejo de desechos. Estos criterios fueron considerados como primordiales al momento de resolver el problema de ubicación de las papeleras en la zona urbana de estas ciudades.

En Ecuador, López et al. (2019, julio 24-26) evaluaron la gestión de almacenamiento de un almacén de bienes de consumo que tenía problemas como demasiado tiempo para realizar operaciones o una gran movilización de personas y productos. Los autores presentaron una propuesta de mejora para reducir los tiempos y costos de preparación de los pedidos a través de la aplicación de un modelo de programación de enteros binarios. Herrera-Granda et al. (2019) realizaron la optimización de la red de contenedores de residuos sólidos urbanos de Ibarra a través de un modelo de programación multiobjetivo de enteros mixtos.

En Chile Banguera et al. (2018) desarrollaron un modelo de programación lineal de enteros mixtos para ubicar instalaciones capacitadas, las cuales cumplen con los objetivos de reciclaje y minimizan el costo de las multas en caso de inviabilidad. De esta manera se calculan los flujos óptimos, la configuración de las instalaciones de recolección, reprocesamiento y reciclaje, así como la estimación de la cantidad de dinero necesario para la operación factible del negocio.

\subsection{Tendencias para el futuro}

Las tendencias futuras en general versarán sobre la aplicación conjunta de métodos aplicados en otras áreas creando enfoques híbridos que permitan obtener soluciones más reales y de aplicación práctica para los problemas de la LI, los cuales deben extenderse a varios campos, en 
los cuáles sea factible implementar procesos de Ll; por esta razón, en el 2020, se han publicado algunos artículos que se acoplan con estos nuevos enfoques. Un ejemplo es el trabajo de Zarbakhshnia et al. (2020), quienes proponen un nuevo enfoque híbrido de toma de decisiones con atributos múltiples, influenciado por un proceso de jerarquía analítica difusa y la optimización de objetivos múltiples.

De la misma manera, Govindan et al. (2020) desarrollaron un enfoque híbrido de proceso de red de análisis difuso, laboratorios de prueba y evaluación de toma de decisiones difuso y modelos de programación lineal entera mixta para la selección circular de proveedores y la asignación de pedidos en una cadena de suministro circuito cerrado, uno de los problemas más habituales en LI. Una de las principales conclusiones planteadas por los autores determina que la aplicación del modelo propuesto es rentable y respetuoso con el medio ambiente. Así también, Aydin (2020) desarrolló un modelo matemático estocástico basado en escenarios y el diseño de la red de LI para mejorar la gestión de escombros, considerando la composición de los terrenos.

Otro aspecto que se marca como tendencia de estudio para el futuro corresponde a la economía circular y el compromiso de recuperación de los productos como estrategia para recuperar valor. Jin et al. (2020) analizaron el restablecimiento de valor a través de la recuperación de unidades de disco duro y determinaron que la mejor manera de contribuir al medio ambiente es reutilizando dichas unidades $y$, como segunda alternativa, se debería aplicar un proceso de recuperación del conjunto de imanes o de imán a imán. Los autores sugieren para investigaciones futuras realizar una optimización cuyo objetivo sea minimizar la huella ambiental. En este sentido, Donner et al. (2020) presentaron su trabajo en el cual estudian desechos agrícolas y la valoración de subproductos, y proponen seis maneras diferentes de manejar los desechos, a través de una nueva jerarquización de las actividades de LI.

Estos enfoques muestran una nueva tendencia con enfoque en el desarrollo sostenible. Por medio de la utilización de varias herramientas, se puede constatar que las diferentes alternativas para generar una recuperación de los productos y residuos marcan las pautas para obtener beneficios multilaterales dirigigdos a empresas, clientes, personas, comunidades, medio ambiente, gobierno, entre otros grupos de interés. Las tendencias como la economía circular, la LI pura, el reciclaje, la recuperación y tratamiento de residuos, entre otros, deben ser analizadas desde el punto de vista económico y social para determinar los beneficios reales que generan para todos los involucrados.

\section{Conclusiones}

Una de las primeras conclusiones después la revisión de la literatura está relacionada con el ámbito de aplicación de los estudios desarrollados. Los modelos matemáticos para la optimización y diseño de la cadena de suministro inversa son los temas que más llaman la atención de los investigadores, porque son los métodos que mayor exactitud brindan para crear soluciones con miras a resolver problemas de empresas y productos que por su naturaleza necesitan ser devueltos a su lugar de origen. En este sentido, según se explica en la sección de tendencias al futuro, es necesario plantear estudios que contemplen en sus modelos matemáticos y de acción, variables de carácter económico, ambiental y social, es decir, considerar todos estos componentes para evaluar de mejor manera el impacto y las repercusiones que puede tener realizar o no la LI. 
Este trabajo sirvió para analizar el campo de aplicación de la LI y se constató que la mayoría de los análisis fueron hechos en Estados Unidos, Europa o países asiáticos, mientras que en América Latina no existe el suficiente sustento científico ni experimentación de campo que brinde resultados concluyentes. Existen primeras aproximaciones llevadas a cabo en Brasil, Colombia, Venezuela, Argentina y Cuba enfocadas en el diseño de rutas para cadenas de LI, sin embargo, sigue siendo un ámbito que aún requiere de estudio y experimentación profunda, dado que la aplicación a nivel regional continúa siendo empírica, sin crear conciencia empresarial y ciudadana sobre la importancia de hacer actividades de LI. Deberían establecerse políticas públicas que tengan como objetivo fomentar el desarrollo económico y social de los pueblos y comunidades a través de actividades como los procesos de LI.

Es importante recalcar que la LI, en su sentido más integral, se refiere al flujo de retorno, no exclusivamente al de productos, sino a todos los suministros que se emplean en la logística directa, lo que incluye información, conocimiento y productos. Además, la LI no es exactamente proporcional ni en tiempo ni en recursos a la logística directa; es decir que los suministros utilizados en la logística directa retornarán en menos del 50 \% a su lugar de origen; además, la cadena de LI implica muchos más eslabones que la cadena de logística directa, lo que implica la participación de más actores y costos adicionales que deben ser considerados. Adicionalmente, no existen métodos técnicos establecidos para manejar y gestionar los flujos de la LI, de manera que los inventarios, cantidades por ordenar, recibir, entre otros, son manejadas por experiencia de los involucrados sin ser necesariamente los óptimos.

Entre las principales motivaciones de la LI se encuentran la responsabilidad medioambiental y la legislación verde que obliga a las empresas a responsabilizarse de sus productos, incluso una vez terminada su vida útil; no obstante, la LI también es una estrategia comercial para recuperar los productos que por alguna condición no satisfacen al cliente, incluso si están en condiciones óptimas y pueden ser distribuidos de nuevo. Asimismo, los productos que han terminado su vida útil y son recuperados por el productor, pueden contener información interesante para él como modo de uso del producto, tiempo de vida real del producto, condiciones en las cuales se manejan los productos, entre otra información que puede ser utilizada para mejorar las condiciones del producto o las recomendaciones de uso.

Finalmente, se puede indicar de manera general que los temas pendientes por investigar y aportar al conocimiento científico se relacionan con costos ambientales, LI para productos perecederos, valuación de inventarios y cantidades óptimas de productos que deben ser recuperados y su tratamiento; modelos de optimización, procesos de LI y gestión de calidad; tecnología de la información en el éxito de los procesos de LI, entre otros relacionados. No obstante, es necesario ampliar la investigación y aplicación de la LI en América Latina, pues la literatura existente es muy sucinta, lo que impide el establecimiento de un marco de referencia regional sobre la LI.

\section{Agradecimiento}

Este artículo se deriva del Proyecto DIUC "Análisis de la logística inversa y su impacto, aplicado al sector de comercialización de neumáticos, en la provincia del Azuay". Este proyecto fue apoyado por la Dirección de Investigación de la Universidad de Cuenca (DIUC), en el marco de la XVI Convocatoria DIUC para Proyectos de Investigación al I Concurso Universitario de Proyectos de 
Investigación de Asignación Horaria Exclusiva, el cual contó con el apoyo de la Pontificia Universidad Javeriana de Colombia.

\section{Referencias}

Abuabara, L., Paucar-Caceres, A., \& Burrowes-Cromwell, T. (2019). Consumers values and behaviour in the Brazilian coffee-in-capsules market: Promoting circular economy. International Journal of Production Research, 57(1), 1-20. https://doi.org/10.1080/00207543.2019.1629664

Agrawal, S., \& Singh, R. (2019) Analyzing disposition decisions for sustainable reverse logistics: Triple Bottom Line approach. Resources, Conservation and Recycling, 150, artículo 104448. https:// doi.org/10.1016/j.resconrec.2019.104448

Agrawal, S., Singh, R., \& Murtaza, Q. (2016a). Disposition decisions in reverse logistics: Graph theory and matrix approach. Journal of Cleaner Production, 137, 93-104. https://doi.org/10.1016/j.jclepro.2016.07.045

Agrawal, S., Singh, R., \& Murtaza, Q. (2016b). Outsourcing decisions in reverse logistics: Sustainable balanced scorecard and graph theoretic approach. Resources, Conservation \& Recycling, 108, 41-53. https://doi.org/10.1016/j.resconrec.2016.01.004

Agrawal, S., Singh, R., \& Murtaza, Q. (2016c). Prioritizing critical success factors for reverse logistics implementation using fuzzy-TOPSIS methodology. Journal of Industrial Engineering International, 12(1), 15-27. https://doi.org/10.1007/s40092-015-0124-8

Alamerew, Y., \& Brissaud, D. (2020). Modelling reverse supply chain through system dynamics for realizing the transition towards the circular economy: A case study on electric vehicle batteries. Journal of Cleaner Production, 254, artículo 120025, https://doi.org/10.1016/j.jclepro.2020.120025

Amin, S., \& Baki, F. (2017). A facility location model for global closed-loop supply chain network design. Applied Mathematical Modelling, 41, 316-330. https://doi.org/10.1016/j.apm.2016.08.030

Amin, S., Zhang, G., \& Akhtar, P. (2017). Effects of uncertainty on a tire closed-loop supply chain network. Expert Systems with Applications: An International Journal, 73(Issue C), 82-91. https://doi. org/10.1016/j.eswa.2016.12.024

Aydin, N. (2020). Designing reverse logistics network of end-of-life-buildings as preparedness to disasters under uncertainty. Journal of Cleaner Production, 256, artículo 120241. https://doi. org/10.1016/j.jclepro.2020.120341

Banguera, L., Sepúlveda, J., Ternero, R., Vargas, M., \& Vásquez, O. (2018). Reverse logistics network design under extended producer responsibility: The case of out-of-use tires in the Gran Santiago city of Chile. International Journal of Production Economics, 205I, 193-200. https://doi.org/10.1016/j. ijpe.2018.09.006

Batarfi, R., Jaber, M., \& Aljazzar, S. (2017). A profit maximization for a reverse logistics dual-channel supply chain with a return policy. Computers \& Industrial Engineering, 106, 58-82. https://doi. org/10.1016/j.cie.2017.01.024

Bouzon, M., Govindan, K., \& Rodriguez, C. (2018). Evaluating barriers for reverse logistics implementation under a multiple stakeholders' perspective analysis using grey decision making approach. Resources, Conservation and Recycling, 128, 315-335. https://doi.org/10.1016/j.resconrec.2016.11.022

Bustos F. C. (2015). La logística inversa como fuente de producción sostenible. Actualidad Contable Faces, 18(30), 7-32. https://www.redalyc.org/articulo.oa?id=25739666002

Carter, C., \& Ellram, L. (1998). Reverse logistics: A review of the literature and framework for future investigation. Journal of Business Logistic, 19(1), 85-102. https://bit.ly/3094SEN

Cruz-Netro, Z., Medina, J., Cartas, A., y Garza, R. (2016). Solid waste management in Mexico's offshore platform construction: Determining potential supply for a reverse logistics process. NETNOMICS: 
Economic Research and Electronic Networking, 17(1), 71-94. https://doi.org/10.1007/s11066016-9105-3

De Brito, M., \& Dekker, R. (2014). A framework for reverse logistics. En Dekker R., Fleischmann M., Inderfurth K., Van Wassenhove L.N. (Eds.), Research in Management (pp. 3-27). https://doi. org/10.1007/978-3-540-24803-3_1

De la Hoz, E., Vélez, J., \& López, L. (2017). Modelo de programación lineal multiobjetivo para la logística inversa en el sector plástico de polipropileno. Información Tecnológica 28(5), 31-36. https:// doi.org/10.4067/S0718-07642017000500005

De Oliveira, C., Luna, M., \& Campos, L. (2019). Understanding the Brazilian expanded polystyrene supply chain and its reverse logistics towards circular economy. Journal of Cleaner Production, 235, 562-573. https://doi.org/10.1016/j.jclepro.2019.06.319

De Oliveira, T., \& Borges, A. (2020). The development role of customers in the reverse logistics of industrial waste. En Business, Trade and Institutional Sustainability (245-254). https://doi. org/10.1007/978-3-030-26759-9_57

Dondo, R., \& Méndez, C. A. (2016). Operational planning of forward and reverse logistic activities on multiechelon supply-chain networks. Computers and Chemical Engineering, 88(C), 170-184. https:// doi.org/10.1016/j.compchemeng.2016.02.017

Donner, M., Gohier, R., \& Vries, H. (2020). A new circular business model typology for creating value from agro-waste. Science of the Total Environment, 716, artículo 137065. https://doi.org/10.1016/j. scitotenv.2020.137065

Fattahi, M., \& Govindan, K. (2017). Integrated forward/reverse logistics network design under uncertainty with pricing for collection of used products. Annals of Operations Research 253(1), 193-225. https://doi.org/10.1007/s10479-016-2347-5

García-Rodríguez, F., Castilla-Gutiérrez, C., \& Bustos-Flores, C. (2013). Implementation of reverse logistics as a sustainable tool for raw material purchasing in developing countries: The case of Venezuela. International Journal Production Economics, 141(2), 582-592. https://doi.org/10.1016/j. ijpe.2012.09.015

Gómez Montoya, R., Correa Espinal, A., \& Vásquez Herrera, L. (2017). Logística inversa: Un enfoque con responsabilidad social empresarial. Criterio Libre, 10(16), 144-158. https://doi.org/10.18041/19000642/criteriolibre.16.1167

Govindan, K., Agarwal, V., Dhingra, J., \& Jha, P. (2019). An integrated decision making model for the selection of sustainable forward and reverse logistic providers. Annals of Operations Research, 273(1-2), 607-650. https://doi.org/10.1007/s10479-017-2654-5

Govindan, K., \& Bouzon, M. (2018). From a literature review to a multi-perspective framework for reverse logistics barriers and drivers. Journal of Cleaner Production, 187, 318-337. https://doi. org/10.1016/j.jclepro.2018.03.040

Govindan, K., Fattahi, M., \& Keyvanshokooh, E. (2017). Supply chain network design under uncertainty: A comprehensive review and future research directions. European Journal of Operational Research, 263(1), 108-141. https://doi.org/10.1016/j.ejor.2017.04.009

Govindan, K., Jha, P., Agarwal, V., \& Dhingra. J (2019). Environmental management partner selection for reverse supply chain collaboration: A sustainable approach. Journal of Environmental Management, 236, 784-797. https://doi.org/10.1016/j.jenvman.2018.11.088

Govindan, K., Kadziński, M., Ehling, R., \& Miebs, G. (2019). Selection of a sustainable third-party reverse logistics provider based on the robustness analysis of an outranking graph kernel conducted with ELECTRE I and SMAA. Omega, 85, 1-15. https://doi.org/10.1016/j.omega.2018.05.007

Govindan, K., Mina, H., Esmaeili, A., \& Mohammad, S. (2020). An integrated hybrid approach for circular supplier selection and closed loop supply chain network design under uncertainty. Journal of Cleaner Production, 242, 118317. https://doi.org/10.1016/j.jclepro.2019.118317 
Govindan, K., Paam, P., \& Abtahi, A. (2016). A fuzzy multi-objective optimization model for sustainable reverse logistics network design. Ecological Indicators, 67, 753-768. https://doi.org/10.1016/j. ecolind.2016.03.017

Guarnieri, P., Cerqueira-Streit, J., \& Batista, L. (2020). Reverse logistics and the sectoral agreement of packaging industry in Brazil towards a transition to circular economy. Resources, Conservation \& Recycling, 153, 104541. https://doi.org/10.1016/j.resconrec.2019.104541

Gutberlet, J., Carenzo, S., Kain, J., \& Martiniano, A. (2017). Waste picker organizations and their contribution to the circular economy: Two case studies from a Global South perspective. 6(4), 52, MDPI Resources. https://doi.org/10.3390/resources6040052

Herrera-Granda, W. G., Imbaquingo-Usiña, W., Lorente-Leyva, L., Herrera-Granda, E., Peluffo-Ordoñez, D, Rossit, D. (2019). Optimization of the network of urban solid waste containers: A case study. En Botto-Tobar M., Pizarro G., Zúñiga-Prieto M., D’Armas M., Zúñiga Sánchez M. (Eds), Technology Trends, CITT: Vol 895 (pp. 91-103). Springer. https://doi.org/10.1007/978-3-030-05532-5_44

Heydari, J., Govindan, K., \& Jafari, A. (2017). Reverse and closed loop supply chain coordination by considering government role. Transportation Research Part D: Transport and Environment, 52(parte A), 379-398. https://doi.org/10.1016/j.trd.2017.03.008

Jin, H., Frost, K., Sousa, I., Ghaderi, H., Bevan, A., Zakotnik, M., \& Handwerker, C. (2020). Life cycle assessment of emerging technologies on value recovery from hard disk drives. Resources, Conservation and Recycling, 157, artículo 104781. https://doi.org/10.1016/j.resconrec.2020.104781

Johnson, F., Vega, J., Cabera, G., \& Cabrera, E. (2015). Ant colony system for a problem in reverse logistic. Studies in Informatics and Control, 24(2), 133-140. https://doi.org/10.24846/v24i2y201501

Julianelli, V., Caiado, R., Scavarda, L., \& Pinto, S. (2020). Interplay between reverse logistics and circular economy: Critical success factors-based taxonomy and framework. Resources, Conservation and Recycling, 158, artículo 104784. https://doi.org/10.1016/j.resconrec.2020.104784

Karagoz, S., Aydin, N., \& Simic, V. (2020). End-of-life vehicle management: A comprehensive review. Journal of Material Cycles and Waste Management, 22(2), 416-442. https://doi.org/10.1007/s10163019-00945-y

Kazemi, N., Modak, N., \& Govindan, K. (2019). A review of reverse logistics and closed loop supply chain management studies published in IJPR: A bibliometric and content analysis. International Journal of Production Research, 57(15-16), 4937-4960. https://doi.org/10.1080/00207543.2018. 1471244

Lagos, C., Guerrero, G., Cabrera, E., Moltedo, A., Johnson, F., \& Paredes, F. (2018). An improved particle swarm optimization algorithm for the VRP with simultaneous pickup and delivery and time windows. IEEE Latin America Transactions, 16(6), 1732-1740. https://doi.org/10.1109/TLA.2018.8444393

Li, J., Wang, J., Pan, Q., Duan, P., Sang, H., Gao, K., \& Xue, Y. (2017). A hybrid artificial bee colony for optimizing a reverse logistics network system. Soft Computing. Springer Berlin Heidelberg, 21(20), 6001-6018. https://doi.org/10.1007/s00500-017-2539-1

Liu, W., Wan, Z., Wan, Z., \& Bing, G. (2019). Sustainable recycle network of heterogeneous pharmaceuticals with governmental subsidies and service-levels of third-party logistics by bi-level programming approach. Journal of Cleaner Production, 249, artículo 119324. https://doi.org/10.1016/j.jclepro.2019.119324

López, J., Freile, Á., \& Iglesias, S. (2019, julio 24-26 ). Reduction in picking times orders for delivery in a national distribution center: A case study. 17 th LACCEI International Multi-Conference for Engineering, Education, and Technology: "Industry, Innovation, and Infrastructure for Sustainable Cities and Communities" Montego Bay, Jamaica. https://doi.org/10.18687/LACCEI2019.1.1.366

Mahmoudi, M., \& Parviziomran, I. (2020). Reusable packaging in supply chains: A review of environmental and economic impacts, logistics system designs, and operations management. International Journal of Production Economics, 228(3), artículo 107730. https://doi.org/10.1016/j. ijpe.2020.107730 
Mangla, S., Govindan, K., \& Luthra, S. (2016). Critical success factors for reverse logistics in Indian industries: A structural model. Journal of Cleaner Production, 129, 608-621. https://doi. org/10.1016/j.jclepro.2016.03.124

Mar, J., \& Gracia, M. (2015). Logística inversa: Prácticas actuales, tendencias futuras y oportunidades de investigación. Revista QUID: Investigación, Ciencia y Tecnología, 23(2014), 31-40. https://dialnet.unirioja.es/servlet/articulo?codigo $=5235933$

Mihi-Ramírez, A., Arias-Aranda, D., \& García-Morales, V. (2012). La gestión de la logística inversa en las empresas españolas: Hacia las prácticas de excelencia. Universia Business Review, 33, 70-82. https://www.redalyc.org/articulo.oa?id=43323186004

Misni, F., \& Lee, L. S. (2017). A Review on strategic, tactical and operational decision planning in reverse logistics of green supply chain network design. Journal of Computer and Communications, 5(8), 83-104. https://doi.org/10.4236/jcc.2017.58007

Mukherjee, C., Denney, J., Mbonimpa, E., Slagley, J., \& Bhowmik, R. (2020). A review on municipal solid waste-to-energy trends in the USA. Renewable and Sustainable Energy Reviews, 119, artículo 109512. https://doi.org/10.1016/j.rser.2019.109512

Noé, C. (2015). Relación entre logística inversa y desempeño: Estudio de casos en Córdoba, Argentina. Cuadernos de Administración, 31(53), 85-96. https://bit.ly/3rcgHMm

Oyola, J., \& Amaya-Mier, R. (2019, marzo 5-7). A reverse logistics network optimization model for residual OTR tires from the mining industry: A Colombian case study [Proceedings]. International Conference on Industrial Engineering and Operations Management, Bangkok, Tailandia. https://bit. ly/3g9NRpL

Peña-Montoya, C., Osorio-Gómez, J., Vidal-Holguin, C., Torres-Lozada, P., \& Marmolejo-Rebellon, L. (2015). Reverse logistics in the plastics subsector: Main facilitators and barriers. Ingeniería e Investigación, 35(3), 27-33. https://doi.org/10.15446/ing.investig.v35n3.49834

Peña-Montoya, C., Bouzon, M., Torres-Lozada, P., \& Vidal-Holguin, J. (2020). Assessment of maturity of reverse logistics as a strategy to sustainable solid waste management. Waste Management \& Research: The Journal for a Sustainable Circular Economy, 38(1), 65-76. https://doi. org/10.1177/0734242x19897131

Pérez-Salazar, M., Aguilar, A., Cedillo-Campos, M., \& Hernández, J. (2017). The role of knowledge management in supply chain management: A literature review. Journal of Industrial Engineering Management, 10(4), 711-788. https://doi.org/10.3926/jiem.2144

Piyathanavong, V., Garza-Reyes, J., Kumar, V., Maldonado-Guzman, G., y Mangla, S. (2019). The adoption of operational environmental sustainability approaches in the Thai manufacturing sector. Journal of Cleaner Production, 220, 507-528. https://doi.org/10.1016/j.jclepro.2019.02.093

Rabbani, M., Saravi, N., \& Farrokhi-Asl, H. (2017). Design of a forward/reverse logistics with environmental considerations. International Journals of Supply and Operations Management, 4(2), 129-146. https://doi.org/10.22034/2017.2.02

Ranjbaran, F., Kashan, A., \& Kazemi, A. (2019). Mathematical formulation and heuristic algorithms for optimisation of auto-part milk-run logistics network considering forward and reverse flow of pallets. International Journal of Production Research, 58(2), 1-35. https://doi.org/10.1080/00207543 .2019 .1617449

Reddy, K., Kummar, A., Sarkis, J., \& Kumar, M. (2020). Effect of carbon tax on reverse logistics network design. Computers \& Industrial Engineering, 139, 106184. https://doi.org/10.1016/j.cie.2019.106184.

Reverse Logistic and Sustainability Organization. (2007). RLSC: About RLSC.

Rodrigues, A., Boscov, M., \& Günther, W. (2020). Domestic flow of e-waste in São Paulo, Brazil: Characterization to support public policies. Waste Management, 102(1), 474-485. https://doi.org/10.1016/j. wasman.2019.10.052 
Rossit, D., Toutouh, J., \& Nesmachnow, S. (2020). Exact and heuristic approaches for multi-objective garbage accumulation points location in real scenarios. Waste Management, 105, 467-481. https://doi.org/10.1016/j.wasman.2020.02.016

Rusel, C., \& Tello, B. (2019). Proceedings of the Future Technologies Conference (FTC). En K. Arai, R. Bhatia y S. Kapoor (Eds.), Proceedings of the Future Technologies Conference (FTC) (Vol. 2, pp, 305323). Springer. https://doi.org/10.1007/978-3-030-02683-7

Saffar, M., Shakouri, H., \& Razmi, J. (2015). A new multi objective optimization model for designing a green supply chain network under uncertainty. International Journal of Industrial Engineering Computations, 6(1), 15-32. https://doi.org/10.5267/j.ijiec.2014.10.001

Sepúlveda-Rojas, J., \& Benitez-Fuentes, P. (2016, mayo 10-14). Coordination and return uncertainties in closed loop supply chains. 6th International Conference on Computers Communications and Control (ICCCC), 188-195, Oradea, Rumania. https://doi.org/10.1109/ICCCC.2016.7496759

Sepúlveda, J., \& Banguera, L. (2017). A model for design of a reverse logistics network under extended producer responsibility. 24th International Conference on Production Research, ICPR.

Silva Rodríguez, J. (2017). Diseño de una red de logística inversa: Caso de estudio Usochicamocha-Boyacá. Ingeniería y Ciencia, 13(26), 91-113. https://doi.org/10.17230/ingciencia.13.26.4

Soto, J., Muñoz, J., \& Giesen, R. (2016). How many urban recycling centers do we need and where ? A continuum approximation approach. Transportation Research Procedia, 12, 851-860. https://doi. org/10.1016/j.trpro.2016.02.038

Srivastava, S. (2008). Network design for reverse logistics. Omega: The International Journal of Management Science, 36(4), 2007-2009. https://doi.org/10.1016/j.omega.2006.11.012

Stock, J. (1992). Reverse logistics in the supply chain. Council of Logistics Management.

Tosarkani, B., \& Amin, S. (2018). A multi-objective model to configure an electronic reverse logistics network and third party selection. Journal of Cleaner Production, 198, 662-682. https://doi. org/10.1016/j.jclepro.2018.07.056

Trochu, J., Chaabane, A., \& Ouhimmou, M. (2020). A carbon-constrained stochastic model for eco-efficient reverse logistics network design under environmental regulations in the CRD industry. Journal of Cleaner Production, 245, artículo 118818. https://doi.org/10.1016/j.jclepro.2019.118818

Uriarte-Miranda, M., Caballero-Morales, S., Martinez-Flores, J., Cano-Olivos, P., \& Akulova, A. (2018). Reverse logistic strategy for the management of tire waste in Mexico and Russia: Review and conceptual model. MDPI Sustainability. https://doi.org/10.3390/su10103398

Van Engeland, J., Beliën, J., De Boeck, L., \& De Jaeger, S. (2018). Literature review: Strategic network optimization models in waste reverse supply chains. Omega, 91. https://doi.org/10.1016/j.omega.2018.12.001

Voigt, D., Casarotto, N., Atherino, M., Guerra, T., \& Garbin da Rocha, R. (2019). Performance evaluation of reverse logistics: Opportunities for future research. Sustainability, 17(19). https://doi. org/10.3390/su11195291

Wang, J., Li, H., Lu, H., Yang, H., \& Wang, C. (2020). Integrating offline logistics and online system to recycle e-bicycle battery in China. Journal of Cleaner Production, 247, artículo 119095. https:// doi.org/10.1016/j.jclepro.2019.119095

Zarbakhshnia, N., Wu, Y., Govindan, K., y Soleimani, H. (2020). A novel hybrid multiple attribute decisionmaking approach for outsourcing sustainable reverse logistics. Journal of Cleaner Production, 242, 118461. https://doi.org/10.1016/j.jclepro.2019.118461 